\title{
A Comparative Study of Frequency of Atrial Fibrillation (AF) in Patients Diagnosed With Ischemic Stroke
}

\author{
MUZAMMIL KHALID ${ }^{1}$, M. ASIF NASEER ${ }^{2}$ \\ ${ }^{1}$ FCPS-II, Department of Medicine, PAF Hospital Mushaf, Sargodha \\ ${ }^{2}$ Head of Department of Medicine, PAF Hospital Mushaf, Sargodha \\ Correspondence to Dr. Muzammil Khalid, E Mail: shahnian99@gmail.com ; muzammilamjad313@gmail.com,Cell \# 0300-7668380
}

\begin{abstract}
Aim: To find frequency of atrial fibrillation (AF) in patients diagnosed with ischemic stroke.

Study design: A Cross sectional descriptive study.

Place and duration of study: Department of Medicine, PAF Hospital Mushaf, Sargodha from July 4, 2019 to Jan 4, 2020.

Methods: In this study a total of 260 patients were taken after taking informed consent meeting inclusion criteria from Department of Medicine, PAF Hospital Mushaf, Sargodha. The informed consent was taken from patients or attendants. Basic information like, age, gender and contact details were taken of all selected subjects. After basic clinical investigation, ECG was done for all patients with 24 hours of Ischemic stroke who presented to the selected setting. Atrial fibrillation was as per operational definition. On pre designed proforma, data was collected from all patients.

Results: The mean age of patients was $57.34 \pm 12.82$ years with minimum and maximum age as 20 and 80 years. There were 150(57.7\%) males. According to operational definition 91 (35\%) cases had atrial fibrillation while other $169(65 \%)$ cases did not have atrial fibrillation. The frequency of atrial fibrillation was statistically same when stratified for age), gender, smoking, obesity, hypertension, diabetes mellitus.

Conclusion: The frequency of atrial fibrillation was seen in $35 \%$ of the cases having ischemic stroke. Hence if atrial fibrillation is diagnosed early then ischemic stroke can be prevented.

Keywords: Stroke, risk factors, complications, echocardiography, atrial fibrillation.
\end{abstract}

\section{INTRODUCTION}

Stroke is one of the most common causes of disability and is the leading cause of death worldwide ${ }^{1,2}$. The strokes (approximately $80 \%$ ) are ischemic, although incidence of hemorrhagic stroke compared to ischemic Stroke vary among different people ${ }^{3}$. Ischemic Stroke represents a major problem of atrial fibrillation $(A F)^{4,5}$. The Data from Stroke registration indicates that atrial fibrillation, both unknown and untreated, causes most of these strokes ${ }^{6,7}$. The frequency of AF vary in patients with stroke and is due to the difficulty of detecting silent phase of AF. The awareness of AF can help to detect and provide safety for susceptible patients ${ }^{8}$. The common associated diseases are valvular heart disease, coronary artery disease and cardiomyopathy. The related comorbidities are Diabetes mellitus, Hypertension, Heart failure, Stroke, chronic obstructive pulmonary disease, kidney failure and cognitive disorders $^{9}$. In 2016 a study reported that $6.5 \%$ cases had AF who had ischemic stroke ${ }^{1}$. Another study in 2014 reported very high frequency of AF i.e. $33.4 \%$ were diagnosed with ischemic stroke as well ${ }^{8}$. The current study is designed to find frequency of AF in patients diagnosed with ischemic stroke in our local population by taking cases from 20-80 years. Through current it may be able to find the exact frequency so that in future the related therapeutic strategies can be opted. The ischemic stroke is a infarction (inadequate blood supply) of the brain, spinal cord or retina to a tissue- based classification ${ }^{10}$ and indicates $~ 71 \%$ of all strokes universally. In 1970s, new trials identified that in patients having stroke the primary clinical deficit is

Received on 26-12-2020
Accepted on 23-04-2021

hibernating, hypo perfused, non-functional part of the brain called as ischemic penumbra ${ }^{11}$. This revolutionary discovery made the basis of reperfusion treatments that have changed the outcomes for patients with ischemic stroke. The first positive trial of stroke thrombolysis was published in $1995^{12}$. In ischemic stroke patient the recent treatment options are Intravenous thrombolysis and Endovascular thrombectomy. The intravenous thrombolysis is effective when given within $4.5 \mathrm{~h}$ of commencement of stroke. In certain patients, who have adequate images of brain perfusion can benefit up to $9 \mathrm{~h}$ or after wake-up stroke onset (stroke symptoms that were not present before sleep) ${ }^{13}$. The incidence of stroke increases after 49 years of age in developed countries compared with developing countries ${ }^{14}$. The non-modifiable risk causes for ischemic stroke are genetic factors, age and sex. Atrial fibrillation (AF) is defined as a narrow complex tachycardia (irregularly irregular) lacking of clear $\mathrm{P}$-waves on a standard 12-lead ECG or a rythum strip. The correlation between $\mathrm{AF}$ and stroke also fails Hill's criterion of specificity ${ }^{15}$. The clinical explanations support the hypothesis that stroke may cause $\mathrm{AF}^{16}$. Most of the patients (approximately 20\%) with ischemic stroke have atrial fibrillation before the onset of stroke. The new atrial fibrillation during the hospital admission was documented in another $5.1 \%$. Serial Holter monitors identified $10.7 \%$ with atrial fibrillation after discharge from hospital. The additional testing with external or implantable loop recorders and mobile telemetry noticed an additional 16.9 $\%$. At the beginning of $20^{\text {th }}$ century, autopsy studies connected AF, mitral stenosis and intra cardiac thrombus formation, so the contribution of $\mathrm{AF}$ in stroke risk was 
identified ${ }^{17}$. Subsequent attempt to quantify the risk of ischemic stroke in each patient develops a scoring system based on current guidelines ${ }^{18}$. The CHA2DS2VASc system was established, by combining various risk factors and minimum age limits of 65 years while additional score is given over age 75 years ${ }^{19}$. This updated score actually has limits: it exclude few risk factors like obstructive sleep apnoea, cardiovascular biomarkers and cerebrovascular diseases biomarkers ${ }^{20}$. The fascinating point is that CHADS and CHA2DS2VASc scores are sum- ups of cardiovascular risk factors. Even in patients without $A F$, both scores are associated with vascular dysfunction measures and are moderately effective in predicting ischemic stroke, myocardial infarction and cardiovascular mortality ${ }^{21}$. The two scores likewise anticipate new beginning of AF, with c-statistics of 0.72 and 0.74 , respectively. Routine investigations for atrial fibrillations, will be a standard ECG on admission, telemetry of admitted patients lasting 12 to $72 \mathrm{~h}$ and, if no other alternative method, further monitoring outpatients, usually at least 1-7 days $^{22}$. Long-term monitoring trials have provided higher recovery rates in randomized patients with cryptogenic stroke. MRI brain having patterns of acute infarction is related with cardio embolism ${ }^{23}$.

Prediction of AF detection after a stroke is made more difficult because some risk factors associated with incident $\mathrm{AF}$, such as diabetes mellitus, are more closely associated with non-cardio embolic ischemic stroke. The presence of atrial runs (lasting < $30 \mathrm{sec}$ ) and excessive supraventricular ectopic activity are associated with an increased probability of $\mathrm{AF}$ detection after a stroke. In patients with AF detected during monitoring and an absolute contraindication to OAC, left atrial appendage occluders could be considered. Although their role and benefit in secondary stroke prevention is not defined.

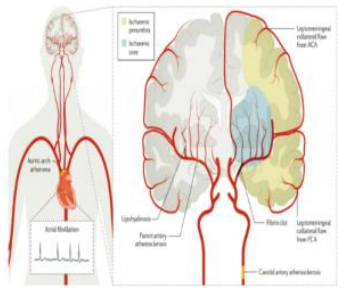

\section{PATIENTS AND METHODS}

This cross-sectional descriptive study had been carried out at Department of Medicine, PAF hospital Mushaf, Sargodha from July 4, 2019 to Jan 4, 2020. All the newly diagnosed cases of ischemic stroke (within 24 hours) of both gender and age between 20-80 years were included in the study. Cases with head injury, Histroy of vasculitis and sinus thrombosis and previous ischemic or haemorrhagic stroke (on available record) are excluded from the study. The informed consent was taken from patients or attendants and ECG was done for all patients with $24 \mathrm{~h}$ of Ischemic stroke who presented to the selected setting. SPSS version 22 was used for data entry and analysis. The Standard deviation and Mean values were calculated for quantitative variables i.e age, while frequency along with percentages are calculated for qualitative variables like gender, AF etc. The familiar Chi-square test was applied to check the relationship with age, gender, smoking, obesity and hypertension. It is considered,Ap-value $<0.05$ as significant.

\section{RESULTS}

Total 260 patients were involved in this study according to inclusion criteria. Average age of the patients was $57.34 \pm$ 12.82 years by age range of 20 to 80 years(Table-1). There were $150(57.7 \%)$ male, with higher male to female ratio. Eighty patients (30.8\%) were smokers while 63 (24.2\%) were obeses and sixty one $(23.5 \%)$ cases were hypertensive.

Table-1: Comparison of frequency of atrial fibrillation w.r.t gender, smoking and BMI (Diabetes mellitus and Hypertension)



Figure-1: Frequency of atrial fibrillation $(n=260)$

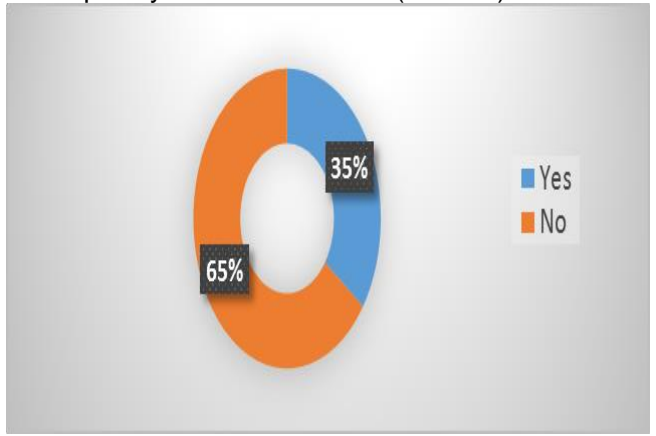

According to operational definition $91(35 \%)$ cases had atrial fibrillation while other $169(65 \%)$ cases did not have atrial fibrillation. Association with atrial fibrillation was significant with age $(p=891)$, gender $(p=0.236)$, smoking 
$(p=0.573)$, Obesity (0.773), Diabetes $(p=0.344)$ and hypertension $(\mathrm{p}=0.914)$.

\section{DISCUSSION}

Atrial fibrillation (AF) is an independent predictor of recurrence and severity of ischemic stroke. World Health Organization define stroke as a clinical disease of speedily growing clinical signs of focal or global (in case of coma) disorders of cerebral function for more than $24 \mathrm{~h}$ or foremost to death with no apparent cause other than a simply vascular origin. The atrial fibrillation (AF) is a very common cardiac arrhythmia in adults which escalate the risk of stroke and related death. In current study 91(35\%) cases had atrial fibrillation while other $169(65 \%)$ cases did not have atrial fibrillation. Though a local study is done in similar context but it was done in elderly patients of ischemic stroke, so they reported frequency of $\mathrm{AF}$ as $41.48 \%{ }^{4}$. The findings of current study is comparable with this study. In 2016 a study reported that $6.5 \%$ cases had AF who had ischemic stroke ${ }^{1}$. In current study we found higher statistics. Another study was done in 2014 and reported very high frequency of AF i.e. $33.4 \%$ were diagnosed with ischemic stroke as well ${ }^{8}$. The findings of this study are similar to current study.

Recent study relates AF frequency when hospitalizations for ischemic, hemorrhagic and a nonstrokes conditions by using inpatient and outpatient. The annual post discharge AF incidence was $3.4 \%(95 \% \mathrm{Cl}$, $3.1 \%-3.7 \%)$ after ischemic stroke, $2.2 \%(95 \% \mathrm{Cl}, 1.9 \%-$ $2.4 \%)$ after hemorrhagic stroke, and $2.9 \%(95 \% \mathrm{Cl}, 2.6 \%-$ $3.1 \%$ ) after non stroke hospitalization. Thus, the study concluded, new AF diagnoses were more common after getting hospitalization for ischemic stroke than for hemorrhagic stroke or other non-stroke conditions. In another study. The rate of AF-related ischemic stroke concluded the previous decade in South Korea and use of preventive antithrombotic treatment before stroke in the national group. The annual percentage of patients belonging to AIS with AF steadily increased from $13.4 \%$ to $22.6 \%$ over the study time frame ( $p$ for patterns $<0.001$ ). Though, the number of patients treated with antiplatelet drugs had increased from $18.8 \%$ in 2004 to $45.3 \%$ in 2013 , while that of patients taking no antithrombotic drugs dropped from $64.6 \%$ in 2004 to $43.9 \%$ in 2013. As a restriction, no data was accessible about non- vitamin $\mathrm{K}$ antagonist, were used in late 2014 in Korea. Studies show, there is a vast gap between clinical practice and guidelines of treatment for patients with AF.

In 2009, one more study was performed to define the frequency at which atrial fibrillation is detected in units. The study was done with 465 patients, with monitoring in the unit of stroke for an average of 54-55 h. The AF was detected in $33(48.5 \%)$ had paroxysmal atrial fibrillation and $51.5 \%$ had persistent $\mathrm{AF}$ ). As the concentrated common risk factor was hypertension, anticoagulation therapy was started in $57.5 \%$ patients. It is concluded, practice of cardiac monitoring in the unit was useful for detecting $A F$ in patients with acute stroke. The result has showed that existence of $\mathrm{AF}$ at stroke beginning and during the acute phase was confirmed by a normal ECG in $869(24.6 \%)$ of 3530 patients with under study ischemic stroke. The patients devoid of arrhythmia and those with AF were more commonly women, of age group 80 years or more, with coronary and peripheral arterial diseases. At the multivariate Cox regression analysis, AF was an independent factor of 30 days and one year mortality ratio, including $17 \%$ of all deaths were by reason of $\mathrm{AF}$. The study has concluded that a higher incidence of AF in patients with a first-ever ischemic stroke, particularly among staged women group.

Likewise another study was done 150 (one hundred and fifty) consecutive patients with ischemic acute stroke placed on 48-hour telemetry monitoring. Among them only thirty five patients had found AF-related strokes. There were 12 patients with AF stroke related, with no previous AF history and were diagnosed with AF stroke. The 6 of the 12 patients were diagnosed with AF at their EKG admission in unit. The remaining six patients had normal entry EKGs and were found to have only AF during telemetry monitoring for $48 \mathrm{~h}$. Therefore, research suggests that $\mathrm{AF}$ is sometimes not diagnosed till stroke develops. The improved AF detection methods are needed for patients at high risk and for primary prevention of stroke. The patients above 65 years of age with non-lacunar stroke should place $48 \mathrm{~h}$ of telemetry monitoring to detect previously undiagnosed $\mathrm{AF}^{24}$. Therefore, it is conclude that all patients with stroke should be investigated with cardiac monitoring for at least $24 \mathrm{~h}$ to find $\mathrm{AF}$ and Patients with elderly age group (>60 years) should be subjected to longer duration.

\section{CONCLUSION}

The frequency of atrial fibrillation was seen in 35\% of the cases having ischemic stroke. Hence if atrial fibrillation is diagnosed early then ischemic stroke can be prevented. Moreover, in patients with ischemic stroke must also be ruled out for atrial fibrillation, with early identification of atrial fibrillation these patients can be managed accordingly. This surly help to have good prognosis if additionally treated.

Conflict of Interest: The authors declare that the research was conducted in the absence of any commercial or financial relationships that could be construed as a potential conflict of interest.

Acknowledgements: The author would like to thank Gp Captain Dr. Muhammad Asif Naseer (Head of Medicine Department at PAF, Hospital, Mushaf). His efforts and valuable comments helped us out in performing and interpreting this study. I also extend our thanks to OC, PAF, Mushaf, Sargodha for providing us a necessary facilities to conduct this study in smooth manner.

\section{REFERENCE}

1. Aziz ZA, Sidek NN, Ngah BA, Looi I, Hanip MR, Basri HB. Clinical Characteristics of Atrial Fibrillation in First-ever Ischemic Stroke Patients; Results from Malaysia National Neurology Registry. Int J Cardiovasc Res. 2016;5(5):doi:10.4172/2324-8602.1000278.

2. Sporns PB, Hanning U, Schwindt W, Velasco A, Minnerup J, Zoubi $T$, et al. Ischemic Stroke. Stroke. 2017:doi.org/10.1161/STROKEAHA.117.016590.

3. Boehme AK, Esenwa C, Elkind MSJCr. Stroke risk factors, genetics, and prevention. 2017;120(3):472-95. 
4. Durrani K, Rasheed M. Atrial fibrillation and ischemic stroke: a hospital based study on elderly patients in Karachi, Pakistan. Khyber Med Uni J. 2012;4(4):193-6.

5. Cotte FE, Chaize G, Kachaner I, Gaudin AF, Vainchtock A, Durand-Zaleski I. Incidence and cost of stroke and hemorrhage in patients diagnosed with atrial fibrillation in France. J Stroke Cerebrovasc Dis. 2014;23(2):8.

6. Freedman B, Potpara TS, Lip GYH. Stroke prevention in atrial fibrillation. The Lancet. 2016;388(10046):806-17.

7. Sposato LA, Cipriano LE, Saposnik G, Vargas ER, Riccio PM, Hachinski V. Diagnosis of atrial fibrillation after stroke and transient ischaemic attack: a systematic review and meta-analysis. Lancet Neurol. 2015;14(4):377-87.

8. Friberg L, Rosenqvist $M$, Lindgren A, Terént A, Norrving $B$, Asplund K. High prevalence of atrial fibrillation among patients with ischemic stroke. Stroke. 2014;45(9):2599-605.

9. Zoni-Berisso M, Lercari F, Carazza T, Domenicucci S. Epidemiology of atrial fibrillation: European perspective. Clinic Epidemiol. 2014;6:213-20.

10. Collaborators GLRoS. Global, regional, and country-specific lifetime risks of stroke, 1990 and 2016. N Engl J Med. 2018;379(25):2429-37.

11. Astrup J, Siesjö BK, Symon L. Thresholds in cerebral ischemia-the ischemic penumbra. Stroke. 1981;12(6):723-5.

12. Stroke TPAfAl. The national institute of neurological disorders and stroke rt-PA stroke study group. N Engl J Med. 1995;333(24):1581-7.

13. Campbell BC, Ma H, Ringleb PA, Parsons MW, Churilov L, Bendszus $\mathrm{M}$, et al. Extending thrombolysis to 4. 5-9 h and wake-up stroke using perfusion imaging: a systematic review and meta-analysis of individual patient data. Lancet. 2019;394(10193):139-47.

14. Feigin VL, Krishnamurthi RV, Parmar P, Norrving B, Mensah $\mathrm{GA}$, Bennett DA, et al. Update on the global burden of ischemic and hemorrhagic stroke in 1990-2013: the GBD 2013 study. Neuroepidemiology. 2015;45(3):161-76.

15. Campbell BC, De Silva DA, Macleod MR, Coutts SB, Schwamm LH, Davis SM, et al. Ischaemic stroke. Nat Rev Dis Primers. 2019;5(1):1-22.
1. 16 Chen P-S, Chen LS, Fishbein MC, Lin S-F, Nattel S. Role of the autonomic nervous system in atrial fibrillation: pathophysiology and therapy. Circ Res. 2014;114(9):1500-15.

16. Hay WE, Levine SA. Age and auricular fibrillation as independent factors in auricular mural thrombus formation. Am Heart J. 1942;24(1):1-3.

17. Wolf PA, Dawber TR, Thomas HE, Kannel WB. Epidemiologic assessment of chronic atrial fibrillation and risk of stroke: The fiamingham Study. Neurology. 1978;28(10):973-.

18. Lip GY, Nieuwlaat R, Pisters R, Lane DA, Crijns HJ. Refining clinical risk stratification for predicting stroke and thromboembolism in atrial fibrillation using a novel risk factor-based approach: the euro heart survey on atrial fibrillation. Chest. 2010;137(2):263-72.

19. Wilson D, Ambler G, Shakeshaft C, Brown MM, Charidimou A, Salman RA-S, et al. Cerebral microbleeds and intracranial haemorrhage risk in patients anticoagulated for atrial fibrillation after acute ischaemic stroke or transient ischaemic attack (CROMIS-2): a multicentre observational cohort study. Lancet Neurol. 2018;17(6):539-47.

20. Chan Y-H, Yiu K-H, Lau K-K, Yiu Y-F, Li S-W, Lam T-H, et al. The CHADS2 and CHA2DS2-VASc scores predict adverse vascular function, ischemic stroke and cardiovascular death in high-risk patients without atrial fibrillation: role of incorporating PR prolongation. Atherosclerosis. 2014;237(2):504-13.

21. Powers WJ, Rabinstein AA, Ackerson T, Adeoye OM, Bambakidis NC, Becker K, et al. 2018 guidelines for the early management of patients with acute ischemic stroke: a guideline for healthcare professionals from the American Heart Association/American Stroke Association. Stroke. 2018;49(3):e46-e99.

22. Best JG, Bell R, Haque M, Chandratheva A, Werring DJ. Atrial fibrillation and stroke: a practical guide. Practical Neurol. 2019;19(3):208-24.

23. Bansil S, Karim H. Detection of atrial fibrillation in patients with acute stroke. J Stroke Cerebrovasc Dis. 2004;13(1):125 